\title{
Prognostic Importance of Vitamins A, E and Retinol-binding Protein 4 in Renal Cell Carcinoma Patients
}

\author{
ROMAN SOBOTKA ${ }^{1}$, OTAKAR ČAPOUN ${ }^{1}$, MARTA KALOUSOVÁ ${ }^{2}$, TOMÁŠ HANUŠ ${ }^{1}$, \\ TOMÁŠ ZIMA ${ }^{2}$, MILADA KOŠTÍŘOVÁ ${ }^{2}$ and VIKTOR SOUKUP ${ }^{1}$ \\ ${ }^{1}$ Department of Urology, General University Hospital and 1st Faculty of Medicine, \\ Charles University, Prague, Czech Republic; \\ ${ }^{2}$ Institute of Medical Biochemistry and Laboratory Diagnostics, \\ General University Hospital and 1st Faculty of Medicine, Charles University, Prague, Czech Republic
}

\begin{abstract}
Aim: To assess the prognostic importance of serum levels of retinol, retinol-binding protein 4 (RBP4) and vitamin $E$ at the time of diagnosis in patients with renal cell carcinoma (RCC). Patients and Methods: In this prospective study, in a cohort of 102 renal cell carcinoma patients, relationships between serum levels of the aforementioned markers and recurrence-free survival (RFS), overall survival (OS), as well as cancer-specific survival (CSS), were evaluated. The vitamin $A$ and vitamin $E$ levels were determined by high-performance liquid chromatography (HPLC), while the RBP4 level by enzyme-linked immunosorbent assay (ELISA). Results: The median followup period was 39 months. Renal cell carcinoma recurred in 9 patients; 23 patients died with 12 of them from RCC. The preoperative vitamin E level was associated to RFS ( $p=0.02)$. We found a significant relationship between $O S$ and the level of RBP4 ( $p=0.002)$, retinol $(p=0.037)$ and vitamin $E$ $(p=0.007)$. The CSS period was significantly associated with the level of RBP4 ( $p=0.0001)$ and retinol $(p=0.0003)$. Patients with an RBP4 level less than $21.0 \mathrm{mg} / \mathrm{l}$ at the time of diagnosis had a 13.5-times higher risk of death due to RCC progression; this risk was up to 7.7-times higher with vitamin A levels under $0.52 \mathrm{mg} / \mathrm{l}$. Conclusion: Low levels of vitamin $A, E$ and RBP4 at the time of RCC diagnosis are associated with a poorer prognosis after surgery.
\end{abstract}

Correspondence to: Dr. Victor Soukup, Department of Urology, General Teaching Hospital and 1st Faculty of Medicine, Charles University in Prague, Ke Karlovu 6, Prague 2, 12808, Czech Republic. Tel: +420 607950393. Fax: +420 224967102, e-mail: viktor.soukup@seznam.cz

Key Words: Renal cell carcinoma, prognosis, vitamin A, vitamin E, retinol-binding protein, overall survival, disease-specific survival, recurrence-free survival.
Renal cell carcinoma (RCC) is the third most common urogenital malignancy in adults with 235,000 new cases recorded globally in 2015 (1). The Czech Republic has the highest RCC incidence in the world (1). The disease is metastatic in up to $20 \%$ of patients at the time of diagnosis. Up to one third of patients relapse during follow-up after radical surgery. The mortality rate is approximately one third of the incidence rate (11 patients per 100,000 inhabitants) (2). Laboratory markers to predict the biological behaviour of renal tumours, the clinical stage of the disease and the success of surgical treatment with regards to the disease-free, disease-specific and overall survival of RCC patients are currently missing.

The subject and etiopathogenesis of RCC have been studied in many significant studies recently. However, the connection of vitamin levels and some nutrition factors with cancer development and their potential prognostic value have not been sufficiently evaluated.

One of the possible factors contributing to RCC development is obesity and the dysregulated production of adipokines, which also affect angiogenesis and insulin resistance development (3, 4). In addition to leptin, tumour-necrosis factor (TNF)-alpha, resistin, galanin and adiponectin, the effect of retinol-binding protein 4 (RBP4) has also recently been mentioned with link to insulin resistance. This protein is produced by adipose cells and affects the negative feedback regulation of glucose transporter type 4, which leads to a decreased insulin sensitivity in fat and other tissues (5). Insulin-like growth factor-1 (IGF-1), which is also a prognostic RCC factor at the time of diagnosis, may be a potential mediator between obesity and RCC development (6). However, the relationship between RBP4 and RCC has not been sufficiently tested.

Oxidative stress and reactive forms of oxygen and nitrogen cause lipid peroxidation, which leads to carcinogenic aldehydes speeding up tumorigenesis (7). Antioxidants, such as enzymes, vitamins, selenium, 
melatonin or glutathione, prevent tumour development. Vitamin A has been found to be able to reduce the incidence of cancers caused by carcinogenic polycyclic aromatic hydrocarbons (8). In blood, vitamin A is transported bound to plasma RBP4. The plasma RBP4 level is indicative of vitamin A tissue bioavailability. Urine RBP4 level reflects the function of proximal tubules and is considered the most sensitive biomarker of tubular damage (9). Clear cell renal cell carcinoma (ccRCC) arises from the proximal tubule. However, there are no reports of relationship between RBP4 and ccRCC.

Vitamin $\mathrm{E}$ has also been assessed in various studies for its potential influence on the risk of urological malignancies (10). Currently, there is no report determining the relationship between vitamin $\mathrm{E}$ and renal tumours.

The primary study objective is to evaluate the relationships of preoperative vitamin A, E and RBP4 levels with recurrence-free survival (RFS), overall survival (OS) and cancer-specific survival (CSS) with RCC patients. The secondary study objective is to evaluate the relationships of preoperative vitamin A, E and RBP4 levels with clinical and pathological prognostic factors of RCC.

\section{Patients and Methods}

Patients' selection and study procedures. A total of 115 patients with renal tumours were enrolled in the study from September 2011 to March 2013, including patients with benign kidney tumours. Abdominal computed tomography (CT) and chest X-ray/CT were performed in all patients. Radical nephrectomy (RN) was carried out in 62 patients and partial nephrectomy (PN) in 51. Tumour biopsy was performed in two cases before systemic therapy. The exclusion criteria were: a history of another tumour (except for nonmelanoma skin tumours), creatinine $>200 \mu \mathrm{mol} / 1$, alanine transaminase (ALT) and/or aspartate transaminase (AST) $\geq 5$ times the upper limit of normal, fasting blood glucose $>15 \mathrm{mmol} / \mathrm{l}$, haemoglobin $<70 \mathrm{~g} / 1$. Blood samples were retrieved from all patients on the morning of surgery. The tumour size, grade and stage were determined by histological examination. After surgery, patients underwent a prospective follow-up as recommended by the European Association of Urology guidelines (11).

The research project was designed as a prospective observational cohort study approved by the local Ethics Committee in accordance with the Declaration of Helsinki. All study participants signed informed consent forms.

Laboratory analyses. Blood was drawn into anticoagulant-free tubes for special biochemical analyses of vitamin A, vitamin E and RBP4. The blood was centrifuged at $1,450 \times g$ for $10 \mathrm{~min}$ and the serum frozen at $-80^{\circ} \mathrm{C}$. The samples were kept in the dark due to the sensitivity of the analysed vitamins to light.

Vitamin A and E levels were determined by high-performance liquid chromatography (HPLC) with standard ClinRep kits according to the manufacturer's protocol (RECIPE Chemicals + Instruments $\mathrm{GmbH}$, Munich, Germany) with UV-light detection at $325 \mathrm{~nm}$ for vitamin A and $295 \mathrm{~nm}$ for vitamin E. The method was standardized and concords with the external quality control programme (Instand $\mathrm{eV}$ certificate). The level of RBP4 was determined by an enzyme-linked immunosorbent assay (ELISA) (Quantikine; R\&D Systems, Minneapolis, MN, USA).

Statistical analysis. Descriptive statistics were used to describe frequency distributions for categorical data, medians and interquartile ranges for other variables. Survival data for 102 patients with renal cancer were censored at the last date the patient was known to be alive or at the analysis cut-off date, whichever came first. Living patients without confirmed metastases were censored for the RFS analysis. All deaths (irrespective of the primary cause) and proven relapses were defined as a study event. For each study group, the time to study event, i.e. OS, DSS and RFS, were expressed by Kaplan-Meier survival curves and the study event risk (hazard ratio (HR) $+95 \%$ confidence interval $(\mathrm{CI})$ ). The optimum value of study parameters was sought by maximizing the test criterion for the Cox regression model and then for several different cut-off points. Given the distribution of these variables, non-parametric tests (Wilcoxon two-sample test or Kruskal-Wallis test) were used to assess the relationship of the study parameters, i.e. stage, grade of differentiation, metastatic disease, histology and tumour size. The difference in categorical variables among the study groups was tested using the chi-square test. The Spearman's rank correlation coefficient was used to determine the relationships of the study characteristics. Statistical significance was determined at alpha level of $5 \%$. The statistical analysis was performed using SAS 9.4 software (Cary, NC, USA).

\section{Results}

The mean age of patients was 65 years (range $=22-84$ ), with almost three fourths being male. Most patients were overweight with a mean body mass index of 28.2 (range=18.9-38.5). Approximately, one fourth of patients were active smokers with an average consumption of 32 pack years; $27 \%$ of the patients were former smokers and more than $46 \%$ of the patients were non-smokers. Almost $9 \%$ of patients had positive family history of RCC. Three fourths of tumours were found incidentally (74.8\%). In symptomatic patients, the leading symptom was haematuria $(13.9 \%)$. The study cohort comprised of benign, as well as malignant kidney tumours; metastatic RCC was found in nearly $10 \%$ of patients initially. Table I shows the clinical and pathological characteristics of the study cohort. Three fourths of patients underwent open surgery and nearly one half partial nephrectomy.

Follow-up data were available for 102 patients with RCC. One patient died perioperatively and was excluded from the analysis; other patients had benign tumours. The data from these 102 patients were used for survival analyses. Nine patients were lost of follow-up; all of these patients had a localized RCC at the time of surgery and did not relapse until last contact. During the study, with a median of 39 months (range=5-66), 12 patients relapsed. Locoregional relapse occurred in two cases (one finding inoperable, the other patient refused new surgery). Metastatic disease 
Table I. Study group's characteristics.

\begin{tabular}{lrc}
\hline & $\mathrm{N}$ & $\%$ \\
\hline All patients & 115 & 100 \\
Histology & & \\
Benign tumours & 12 & 10.4 \\
Papillary RCC & 11 & 9.6 \\
Chromophobe RCC & 9 & 7.7 \\
Clear RCC & 76 & 66.1 \\
Others & 7 & 6.2 \\
Stage group at time of RCC diagnosis & 103 & \\
Localized & 91 & 88.3 \\
Metastatic & 12 & 11.7 \\
Stage & 103 & \\
pT1 & 58 & 56.3 \\
pT2 & 10 & 9.7 \\
pT3 & 23 & 22.3 \\
pT4 & 12 & 11.7 \\
Grade & 99 & \\
Grade 1 & 19 & 19 \\
Grade 2 & 49 & 49.5 \\
Grade 3 & 22 & 22.5 \\
Grade 4 & 9 & 9 \\
Type of operation & & \\
Nephrectomy & 62 & 54.9 \\
open & & \\
laparoscopic & & \\
Nephron-sparing surgery & & \\
(partial nephrectomy) & 16 & 25.8 \\
open & & \\
laparoscopic & 51 & 45.1 \\
Percutaneous biopsy & 36 & 70.6 \\
\hline & 15 & 29.4 \\
\hline
\end{tabular}

RCC, Renal cell cancer.

occurred in ten patients. A total of 23 patients died, 12 of whom as a result of RCC progression. This is summarized in Table II.

Relationship between vitamin A, E and RBP4 levels and clinicopathological parameters. Table III shows values of study parameters for each tumour stage, differentiation grade, size and each histological sub-type. There were no significant differences in study parameters among the histological RCC sub-groups. The RBP4 level was significantly higher in the primary localized RCC group than in the primarily metastatic group $(p=0.021)$. Vitamin A level was markedly higher in Grade 2 compared to other differentiation grades $(p=0.036)$; however, the number of G2 tumours was more than double compared to other groups. Vitamin E level varied in the localized ccRCC between the pT1 and pT2 category versus pT3 $(p=0.033)$ and was significantly lower in pT3 group.

Survival analyses. As a result of univariate analysis, preoperative vitamin A and RBP4 levels were identified as
Table II. Study group's characteristics.

\begin{tabular}{ll}
\hline No. of patients with localized RCC & $92(88.5 \%)$ \\
No. of patients with metastatic RCC at time of diagnosis & $12(11.5 \%)$ \\
No. of patients with recurrence of RCC & 12 \\
No. of patients alive & 79 \\
No. of patients with non-cancer cause death & 11 \\
No. of patients with cancer cause death & 12 \\
\hline
\end{tabular}

RCC, Renal cell cancer.

significant predictive factors of OS and CSS (Figures 1 and 2). Table IV shows the cut-off values for the tested parameters with statistical significance, HR and $95 \%$ CI for the OS, DSS and RFS.

Patients with RBP4 levels below $21.0 \mathrm{mg} / 1 \mathrm{had}$ a 13.5 times higher risk of cancer-specific mortality $(p=0.002)$, whereas patients with vitamin A levels lower than $0.52 \mathrm{mg} / 1$ had a 7.7-times higher risk of RCC-specific mortality $(p=0.037)$.

Lower values of all study parameters were related to OS. Vitamin E levels less than $15 \mathrm{mg} / 1$ resulted in 3.1-times higher risk of mortality $(p=0.007)$. Vitamin A levels under $0.52 \mathrm{mg} / \mathrm{l}$ and RBP4 levels under $21.0 \mathrm{mg} / 1$ resulted in 2.4times and 4.2-times higher risk of mortality, respectively.

Of the study parameters, only the vitamin E level at the time of surgery was associated with RFS (cut-off $<15 \mathrm{mg} / \mathrm{l}$; $p=0.002, \mathrm{HR}=3.67)$.

\section{Discussion}

Correlations between the preoperative serum vitamin A, E and RBP4 levels and the CSS, OS and RFS in RCC patients were found in this study. Low preoperative RBP4 and vitamin A levels had a negative impact on CSS. RBP4 level was lower in patients with metastatic disease in comparison to the localized RCC group. Additionally, we found that vitamin E level was decreased in higher-stage category of RCC.

Even in the era of modern imaging techniques, there are still patients being diagnosed with advanced and metastatic RCC having poor prognosis. This is due to the asymptomatic course of the disease and the absence of diagnostic markers that are able detect non-invasively and extensively the presence of the disease. Not all factors contributing to the disease development have been identified and there are no reliable prognostic markers that can enable individualized treatment. The significance of vitamins A and E for RCC has not been studied before.

Epidemiological studies show that a long-term limited vitamin A intake may increase the risk of cancer development (12). Under laboratory conditions, the impaired expression of all-trans retinoic acid receptors leads to 


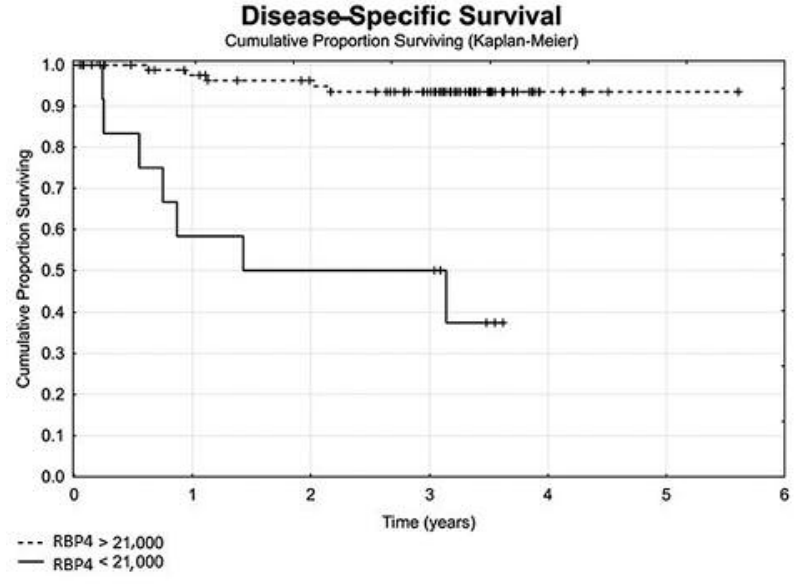

Figure 1. Disease-specific survival of patients with retinol-binding protein 4 (RBP4) level below 21,000 $\mathrm{ng} / \mathrm{ml}$ versus more than or equal to $21,000 \mathrm{ng} / \mathrm{ml}$.

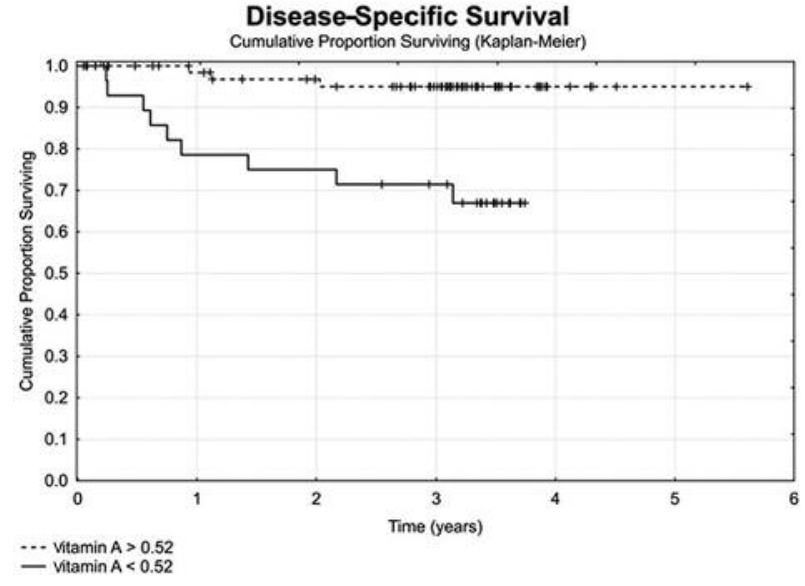

Figure 2. Disease-specific survival of patients with level vitamin $A$ below $0.52 \mathrm{mg} / \mathrm{l}$ versus more than or equal to $0.52 \mathrm{mg} / \mathrm{l}$.

Table III. Associations among vitamin A, E and RBP4 levels with clinical and pathological characteristics.

\begin{tabular}{|c|c|c|c|c|c|c|}
\hline & \multicolumn{2}{|c|}{ Vitamin A } & \multicolumn{2}{|c|}{ RBP4 } & \multicolumn{2}{|c|}{ Vitamin E } \\
\hline & Mean $(m g / l) \pm S D$ & $p$-Value & Mean $(\mathrm{mg} / \mathrm{l}) \pm \mathrm{SD}$ & $p$-Value & Mean $(\mathrm{mg} / \mathrm{l}) \pm \mathrm{SD}$ & $p$-Value \\
\hline Benign tumours $(n=12)$ & $0.64 \pm 0.31$ & 0.508 & $32.4 \pm 16.8$ & 0.160 & $20.3 \pm 7.08$ & 0.992 \\
\hline Localized RCC $(n=91)$ & $0.69 \pm 0.33$ & & $34.5 \pm 11.8$ & & $21.45 \pm 8.58$ & \\
\hline Localized RCC $(n=91)$ & $0.69 \pm 0.33$ & 0.329 & $34.46 \pm 11.82$ & $0.021^{*}$ & $21.45 \pm 8.58$ & 0.853 \\
\hline Metastatic RCC $(n=12)$ & $0.59 \pm 0.32$ & & $25.03 \pm 10.87$ & & $22.6 \pm 10.09$ & \\
\hline Clear RCC $(\mathrm{n}=76)$ & $0.71 \pm 0.35$ & 0.955 & $34.79 \pm 11.39$ & 0.633 & $21.83 \pm 8.78$ & 0.898 \\
\hline Non-clear RCC $(n=20)$ & $0.69 \pm 0.24$ & & $36.59 \pm 11.70$ & & $20.86 \pm 8.04$ & \\
\hline Grade $1(n=19)$ & $0.63 \pm 0.32$ & $0.036^{*}$ & $30.71 \pm 9.38$ & 0.837 & $20.96 \pm 9.72$ & 0.837 \\
\hline Grade $2(n=49)$ & $0.80 \pm 0.39$ & & $35.71 \pm 12.86$ & & $23.61 \pm 9.02$ & \\
\hline Grade $3(n=22)$ & 0.540 .15 & & $32.14 \pm 10.22$ & & $19 \pm 7.95$ & \\
\hline Grade $4(n=9)$ & $0.69 \pm 0.31$ & & $28.95 \pm 12.75$ & & $19.47 \pm 5.61$ & \\
\hline Stage pT1+pT2 $(\mathrm{n}=58)$ & $0.69 \pm 0.34$ & 0.237 & $35.50 \pm 12.20$ & 0.379 & $23.12 \pm 9.24$ & $0033^{*}$ \\
\hline Stage pT3 $(n=18)$ & $0.78 \pm 0.37$ & & $32.51 \pm 8.16$ & & $17.66 \pm 5.47$ & \\
\hline
\end{tabular}

RCC, Renal cell cancer; RBP4, retinol-binding protein 4; SD, standard deviation. *statistically significant difference.

malignant transformation of cell cultures. Animal studies have shown the protective effect of vitamin A in lung, bladder and prostate cancer. Vitamin A also shows high potential for treatment of precancerous skin lesions (13). This study proved that lower serum RBP4 and vitamin A levels at the time of RCC diagnosis are strongly correlated with shorter CSS and OS.

Vitamin A is involved in the proliferation process, vascular endothelial barrier enhancement and participates in immunomodulatory functions. Retinoic acid is also able to prevent cardiac remodelling, as demonstrated in animal models. In addition, inhibition of renin-angiotensin system expression decreases overpressure leading to myocardial hypertrophy. This supports the finding that vitamin A depletion may lead to increased cardiovascular mortality (14). In this study, low vitamin A level and its binding protein shortened OS in RCC patients, which is not only necessarily related to the cancer progression or relapse but may as well be related to many internal co-morbidities, including cardiovascular ones. This may also be connected with the fact that half of the deaths in study cohort (11 out of 23 patients) were not related to RCC progression.

Overall and cardiovascular mortality in haemodialysis patients is associated with low retinol levels (15). Patients with end-stage renal failure often have protein-calorie malnutrition, including trace elements and vitamin deficiency. Reduced retinol bioavailability leads to shorter survival of haemodialysis patients. In this study, more than 
Table IV. Cut-off levels of vitamin A, E and RBP 4 and their relationship to survival parameters.

\begin{tabular}{|c|c|c|c|c|c|c|c|c|c|c|c|c|}
\hline & \multicolumn{4}{|c|}{ OS } & \multicolumn{4}{|c|}{ CSS } & \multicolumn{4}{|c|}{ RFS } \\
\hline & $\mathrm{N}$ & $\begin{array}{c}\mathrm{N} \text { death } \\
(\%)\end{array}$ & $\begin{array}{l}p \text {-Value } \\
\text { (log-rank) }\end{array}$ & $\begin{array}{c}\mathrm{HR} \\
(95 \% \mathrm{CI})\end{array}$ & $\mathrm{N}$ & $\begin{array}{c}\mathrm{N} \text { death } \\
(\%)\end{array}$ & $\begin{array}{c}p \text {-Value } \\
\text { (log-rank) }\end{array}$ & $\begin{array}{c}\mathrm{HR} \\
(95 \% \mathrm{CI})\end{array}$ & $\mathrm{N}$ & $\begin{array}{l}\mathrm{N} \text { death } \\
(\%)\end{array}$ & $\begin{array}{c}p \text {-Value } \\
\text { (log-rank) }\end{array}$ & $\begin{array}{c}\mathrm{HR} \\
(95 \% \mathrm{CI})\end{array}$ \\
\hline Vitamin $\mathrm{A}<0.52 \mathrm{mg} / 1$ & 30 & $11(0.37)$ & $0.037 *$ & $\begin{array}{c}2.388 \\
(1.053-5.414)\end{array}$ & 30 & $9(0.3)$ & $0.002 *$ & $\begin{array}{c}7.758 \\
(2.099-28.677)\end{array}$ & 30 & $6(0.2)$ & 0.950 & $\begin{array}{c}1.031 \\
(0.396-2.683)\end{array}$ \\
\hline Vitamin $A \geq 0.52 \mathrm{mg} / 1$ & 72 & $12(0.17)$ & & & 72 & $3(0.04)$ & & & 72 & $6(0.08)$ & & \\
\hline Vitamin $\mathrm{E}<15 \mathrm{mg} / \mathrm{l}$ & 19 & $9(0.47)$ & $0.007 *$ & $\begin{array}{c}3.152 \\
(1.363-7.291)\end{array}$ & 19 & $4(0.21)$ & 0.130 & $\begin{array}{c}0.407 \\
(0.122-1.355)\end{array}$ & 19 & $8(0.42)$ & $0.002 *$ & $\begin{array}{c}3.67 \\
(1.499-8.988)\end{array}$ \\
\hline Vitamin $E \geq 15 \mathrm{mg} / \mathrm{l}$ & 83 & $14(0.17)$ & & & 83 & $8(0.10)$ & & & 83 & $12(0.14)$ & & \\
\hline $\mathrm{RBP} 4<21.0 \mathrm{mg} / 1$ & 12 & $7(0.58)$ & $0.02 *$ & $\begin{array}{c}3.91 \\
(1.240-12.336)\end{array}$ & 12 & $7(0.58)$ & $0.0001^{*}$ & $\begin{array}{c}13.549 \\
(4.285-42.842)\end{array}$ & 12 & $2(0.17)$ & 0.909 & $\begin{array}{c}0.919 \\
(0.213-3.960)\end{array}$ \\
\hline $\mathrm{RBP} 4 \geq 21.0 \mathrm{mg} / \mathrm{l}$ & 90 & $16(0.18)$ & & & 90 & $5(0.06)$ & & & 90 & $18(0.2)$ & & \\
\hline
\end{tabular}

OS, Overall survival; CSS, cancer-specific survival; RFS, recurrence-free survival; HR, hazard ratio; CI, confidence interval; RBP4, retinol-binding protein $4 . *$ Statistically significant difference.

half of the patients (62 out of 115) underwent a nonnephron sparing surgery. We can, thus, hypothesise that lower vitamin A level before radical nephrectomy is probably associated with long-term protein-calorie malnutrition. This could also explain shorter OS in patients with low vitamin A levels before surgery. Choi et al. published adiponectin and RBP4 availability in 54 RCC patients as a potential biomarker. Contrary to our results, they identified RBP4 levels over $30.27 \mathrm{mg} / \mathrm{l}$ as a risk factor for RCC (16).

With regards to vitamin $\mathrm{E}$, there has been a study of patients with cardiovascular diseases. The supplementation of this vitamin did not prolong OS of patients over 55 years of age at high risk of cardiovascular events because they had cardiovascular disease or diabetes, in addition to one other risk factor. These patients used $400 \mathrm{IU}$ of vitamin $\mathrm{E}$ daily for an average of 4.5 years. High doses may even shorten this survival (17). A meta-analysis published in 2015 proved that there was nearly a $20 \%$ decrease in the risk of RCC incidence in a population using vitamin $\mathrm{E}$ as a food supplement (18). A Canadian study, which was included in the aforementioned meta-analysis, proved an inverse association between the intake of vitamin E, calcium supplements and RCC development in females. In males, the risk of RCC decreased only after 5 years of using vitamin $\mathrm{E}$ and iron supplements (19). Our study showed the effect of lower vitamin E levels at the time of RCC diagnosis on shorter OS and RFS.

To our best knowledge, this is the first study evaluating the relationships between vitamin A, E and RBP4 levels and certain clinical and pathological characteristics of RCC. We are the first to prove the importance of vitamin A and RBP4 as independent predictive factors related to OS and CSS. The association between preoperative vitamin $\mathrm{E}$ levels and RFS is yet to be published.
Our study, however, has certain limitations. It involved a small, heterogeneous patient cohort on which we only carried out one preoperative blood sampling. As a result, the long-term development trends of the study parameter levels are not known. Despite this, vitamin A, E and RBP4 levels were identified as significant prognostic factors for RCC.

\section{Conclusion}

Low vitamin A, E and RBP4 levels at the time of renal cell carcinoma surgery may have an adverse impact on OS. It is evident from our results that RBP4 and vitamin A are important prognostic factors related to DSS. With serum RBP4 levels below $21.0 \mathrm{mg} / \mathrm{l}$ at the time of RCC diagnosis, the risk of RCC mortality is up to 13.5-times higher and, with vitamin A levels below $0.52 \mathrm{mg} / 1$, this risk is up to .7times higher. Low baseline vitamin $\mathrm{E}$ levels increase the risk of RCC relapse.

\section{Acknowledgements}

The Authors would like to thank the nurses from the Department of Urology for help and the laboratory staff for their excellent laboratory skills, especially Ms. Dita Hudcová.

The study was supported by GAUK 135115, BBMRI-CZ LM2015089, Ministry of Health of the CR - DRO VFN64165 and SVV 2002064-2016 grants.

\section{References}

1 http://globocan.iarc.fr (accessed 23 Jan. 2017).

2 www.svod.cz (accessed 23 Jan. 2017).

3 Golabek T, Bukowczan J, Szopinski T, Chlosta P, Lipczynski W, Dobruch $\mathrm{J}$ and Borowka A: Obesity and renal cancer incidence and mortality - A systematic review of prospective cohort studies. Ann Agric Environ Med 23(1): 37-43, 2016. 
4 Wang H, Wu J, Gu W, Wang B, Wan F, Dai B, Zhang H, Shi G, Shen Y, Zhu Y and Ye D: Serum adiponectin level may be an independent predictor of clear cell renal cell carcinoma. J Cancer 7(10): 1340-1346, 2016.

5 Adams-Huet B, Devaraj S, Siegel D and Jialal I: Increased adipose tissue insulin resistance in metabolic syndrome: Relationship to circulating adipokines. Metab Syndr Relat Disord 12(10): 503-507, 2014.

6 Tracz AF, Szczylik C, Porta C and Czarnecka AM: Insulin-like growth factor-1 signalling in renal cell carcinoma. BMC Cancer 16(1): 453, 2016.

7 Barrera G, Gentile F, Pizzimenti S, Canuto RA, Daga M, Arcaro A, Cetrangolo GP, Lepore A, Ferretti C, Dianzani C and Muzio $\mathrm{G}$ : Mitochondrial dysfunction in cancer and neurodegenerative diseases: spotlight on fatty acid oxidation and lipoperoxidation products. Antioxidants 5(1): 7, 2016.

8 Singh R, Sram RJ, Binkova B, Kalina I, Popov TA, Georgieva T, Garte S, Taioli E and Farmer PB: The relationship between biomarkers of oxidative DNA damage, polycyclic aromatic hydrocarbon DNA adducts, antioxidant status and genetic susceptibility following exposure to environmental air pollution in humans. Mutat Res 620(1-2): 83-92, 2007.

9 Ziegelmeier M, Bachmann A, Seeger J, Lossner U, Kratzsch J, Blüher M, Stumvoll M and Fasshauer M: Serum levels of adipokine retinol binding protein-4 in relation to renal function. Diabetes Care 30(10): 2588-2592, 2007.

10 Lippman SM, Klein EA, Goodman PJ, Lucia MS, Thompson IM, Ford LG, Parnes HL, Minasian LM, Gaziano JM, Hartline JA, Parsons JK, Bearden JD 3rd, Crawford ED, Goodman GE, Claudio J, Winquist E, Cook ED, Karp DD, Walther P, Lieber MM, Kristal AR, Darke AK, Arnold KB, Ganz PA, Santella RM, Albanes D, Taylor PR, Probstfield JL, Jagpal TJ, Crowley JJ, Meyskens FL Jr, Baker LH and Coltman CA Jr.: Effect of selenium and vitamin $\mathrm{E}$ on risk of prostate cancer and other cancers: The Selenium and Vitamin E Cancer Prevention Trial (SELECT). JAMA 301(1): 39-51, 2009.
11 http://uroweb.org/guideline/renal-cell-carcinoma/(accessed 23 Jan. 2017).

12 Sun SY and Lotan R: Retinoids and their receptors in cancer development and chemoprevention. Crit Rev Oncol Hematol 41(1): 41-55, 2002.

13 Bushue N and Wan YY: Retinoid pathway and cancer therapeutics. Adv Drug Deliv Rev 62(13): 1285-1298, 2010.

14 Choudhary R, Palm-Leis A, Scott RC, Guleria RS, Rachut E, Baker $\mathrm{KM}$ and Pan J: All-trans retinoic acid prevents development of cardiac remodelling in aortic banded rats by inhibiting the renin-angiotensin system. Am J Physiol Heart Circ Physiol 294(2): 633-644, 2008.

15 Kalousová M, Kubena AA, Kostírová M, Vinglerová M, Ing OM, Dusilová-Sulková S, Tesar V and Zima T: Lower retinol levels as an independent predictor of mortality in long-term haemodialysis patients: A prospective observational cohort study. Am J Kidney Dis 56(3): 513-521, 2010.

16 Choi SH, Chun SY, Kim T-H and Kwon TG: Identifying the emerging role of adipokine as a diagnostic and prognostic biomarker of renal cell carcinoma. Urol Oncol 34(6): 15-19, 2016.

17 Yusuf S, Dagenais G, Pogue J, Bosch J and Sleight P: Vitamin E supplementation and cardiovascular events in high-risk patients. N Engl J Med 342(3): 154-160, 2000.

18 Shen C, Huang Y, Yi S, Fang Z and Li L: Association of vitamin $\mathrm{E}$ intake with reduced risk of kidney cancer: A meta-analysis of observational studies. Med Sci Monit 21: 3420-3426, 2015.

$19 \mathrm{Hu} \mathrm{J}$, Mao Y and White K: Diet and vitamin or mineral supplements and risk of renal cell carcinoma in Canada. Cancer Causes Control 14(8): 705-714, 2003. 